\title{
A Resource Allocation Evolutionary Algorithm for OFDM Based on Karush-Kuhn-Tucker Conditions
}

\author{
Hai-Lin Liu and Qiang Wang \\ School of Applied Mathematics, Guangdong University of Technology, Guangzhou 510520, China \\ Correspondence should be addressed to Qiang Wang; wangqiangzhch@yahoo.cn
}

Received 20 January 2013; Accepted 26 March 2013

Academic Editor: Yuping Wang

Copyright (C) 2013 H.-L. Liu and Q. Wang. This is an open access article distributed under the Creative Commons Attribution License, which permits unrestricted use, distribution, and reproduction in any medium, provided the original work is properly cited.

\begin{abstract}
For orthogonal frequency division multiplexing (OFDM), resource scheduling plays an important role. In resource scheduling, power allocation and subcarrier allocation are not independent. So the conventional two-step method is not very good for OFDM resource allocation. This paper proposes a new method for OFDM resource allocation. This method combines evolutionary algorithm (EA) with Karush-Kuhn-Tucker conditions (KKT conditions). In the optimizing process, a set of subcarrier allocation programs are made as a population of evolutionary algorithm. For each subcarrier allocation program, a power allocation program is calculated through KKT conditions. Then, the system rate of each subcarrier allocation program can be calculated. The fitness of each individual is its system rate. The information of optimizing subcarrier and power allocation can be interacted with each other. So, it can overcome the shortcoming of the two-step method. Computer experiments show the proposed algorithm is effective.
\end{abstract}

\section{Introduction}

Orthogonal frequency division multiplexing (OFDM) has attracted more and more research interest because it is a potential solution for high-rate data service demands [1]. In OFDM system, the high speed data flow is divided into a lot of slow speed data flows. So, the inters symbol interference (ISI) can be reduced greatly. Meanwhile different subcarrier's channel fading condition is independent, so, the OFDM resource allocation has high flexibility. The flexibility of multiuser access can also be improved. The principle of OFDM is described in Figure 1.

The resource allocation problems in OFDM system have two different types of analytical perspectives: one is to maximize the system capacity (RA) when the emission power is limited; the other is to minimize the transmit power (ME) under the condition that the system capacity is limited. However, in the fourth-generation mobile communication system, the main business is the users experience. Therefore, the former perspective causes more attention.

Resource allocation algorithms can be classified into two categories: (a) the model can be solved directly. However, it may get an exact result; the computational complexity is too high. (b) The solving process is divided into several steps through some executive strategies. Though it cannot get such an exact solution, it is simple and easy to handle. After optimization, the progress shows the proposed solution is of acceptable accuracy. The two-step method is described as an effective algorithm. In a two-step method, we first considerate subcarrier allocation then the power allocation. This method may result in low complexity and fast solution speed.

The recent research on the two-step method focuses on the rules of subcarrier allocation with the purpose to take capacity of system and fairness into account. But most attention is paid to find a good algorithm to optimize the power allocation so as to maximize the system capacity. For the optimization of the power distribution, the main methods are classical deterministic optimization algorithm and intelligent optimization algorithms.

At present, most algorithms for OFDM resource allocation focus on how to improve solution efficiency based on system fairness. Algorithms proposed in [2-5] were based on the gradient information. In early research, the affusion algorithm attracted the attention of most scholars. It can maximize the system but make the system fairness worse. Making use of the gradient information, article [2] 
defined a kind of system throughout increase. For each power allocation, enhanced power was allocated to the user who had the maximum throughout. By introducing the constraint conditions according to the definition of throughout increase, the system can enhance throughout and meet the fairness. In paper [3], a framework of algorithm based on gradient information was presented. The model was expressed as a graph optimization problem, and it resolves the problem with dual decomposition. Considering that OFDM resource allocation model is a nonlinear optimization problem, the literature in [5-10] uses Lagrange method to measure resource allocation. Based on Lagrange method, the literature in [11] introduced a self-adaptive iterative algorithm in order to meet the system fairness. In paper [8], the model was converted into problem in graph theory and was solved with graph theory combined with Lagrange method, which gave a satisfactory result. In paper [9], the model was converted into a convex optimization problem, which could be efficiently solved by Lagrange method. Also, many algorithms were put forward by introducing some specific strategies to deal with OFDM resource allocation problem. In these algorithms, classical or intelligent optimization algorithms did not appear, as presented in the literature in [12-20]. By introducing the concept of pareto efficiency, paper [18] presented the trade-off program between operators and users' service. The literature in [21-27] solved OFDM resource allocation problem with intelligent algorithm which takes the advantages of simple operation and convenient calculation. By introducing weight factors, paper [25] combined transmit power and system capacity to a cost function. A modified PSO algorithm was presented to solve the problem and got good performance. Paper [26] presented a multiobjective optimization model for determining the transmit power and system capacity, respectively, and used NSGA-2 algorithm to solve the problem. Due to the mutual restrictive relationship between transmit power and system capacity, a variable was optimized while the other performed worse. Therefore, it is feasible to use multiobjective algorithm for optimization.

Above, most of the literature is based on two-step method. Firstly, subcarrier is allocated, and then, the model is optimized. The two-step method can significantly reduce the complexity of the algorithm. But because the subcarrier allocation and power allocation are dependent, it is unreasonable to allocate subcarrier and power separately. So, the efficiency of the algorithm is not very high. However, if subcarrier allocation and power allocation are optimized at the same time, the complexity of the algorithm is so high that it does not meet the instantaneous requirements for mobile communication system. Therefore, it is expected to improve the accuracy and efficiency of algorithm if the information of subcarrier allocation and power allocation is able to interact with each other in each iteration.

A hybrid evolutionary algorithm for OFDM resource allocation is proposed in this paper. In this algorithm, the subcarrier and power allocation is optimized by turns. Because the OFDM resource allocation is a hybrid optimization problem, the discrete and continuous variables can be optimized by evolutionary algorithm and KKT conditions, respectively. This algorithm, firstly, generates a group

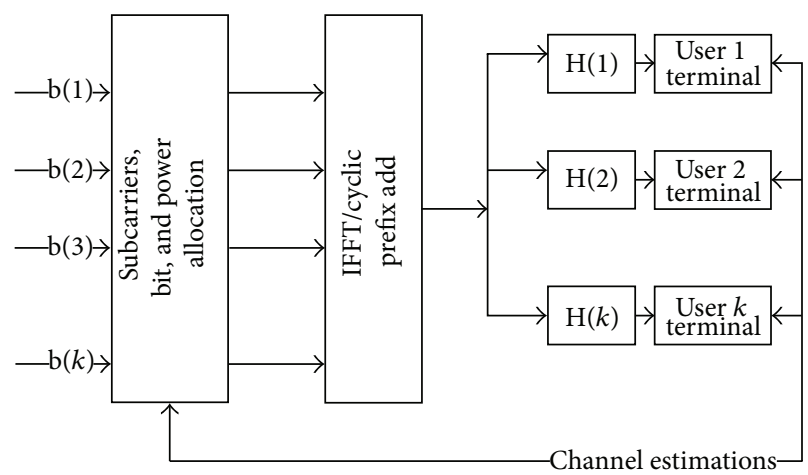

FIgURE 1: System model of the downlink MU-OFDM.

subcarrier allocation program, and then, the corresponding power allocation program is generated by KKT conditions. The system rate of each subcarriers can be obtained. For the evolutionary algorithm, the set of subcarrier is regarded as a population. The system rate is the fitness of each individual. In the optimizing process, the subcarrier and power allocation information can be used with each other. So this algorithm is more efficient.

The remainder of the paper is organized as follows: the model of OFDM resource allocation and algorithm is described at Sections 2 and 3, computer simulation results are shown at Section 4, and conclusions are drawn at Section 5.

\section{The Model of OFDM Resource Allocation}

Considering that spectrum resources are limited, OFDM resource allocation plays an important role in the 4th generation mobile communication system. Each eNodeB has a lot of orthogonal subcarriers allocated to each user. The core problem of OFDM resource allocation is which subcarriers should be allocated to a user and how much each subcarrier's power should be.

Assume that there are $N$ subcarriers shared by $K$ users; define the channel gain of the $k$ th user on the $n$th subcarrier $g_{k, n}$; the noise power is $\sigma^{2}=\left(N_{0} B\right) / N$, where $N_{0}$ is noise power spectral density. The signal-to-noise ratio (SNR) is $h_{k, n}=g_{k, n}^{2} / \sigma^{2}$. The capacity of user $k$ on subcarrier $n$ is normalized by $r_{k, n}=(1 / N) \ln \left(1+\left(p_{k, n} h_{k, n} / \Gamma\right)\right)$, where $\Gamma$ is a constant. So, data rate of user $k$ can be written as

$$
R_{k}=\sum_{n=1}^{N} \frac{w_{k, n}}{N} \ln \left(1+\frac{p_{k, n} h_{k, n}}{\Gamma}\right) .
$$

The mathematic model of resource allocation problem can be described as follows:

$$
\max F(W, P)=\sum_{k=1}^{K} \sum_{n=1}^{N} \frac{w_{k, n}}{N} \ln \left(1+\frac{p_{k, n} h_{k, n}}{\Gamma}\right)
$$




$$
\begin{aligned}
& \text { s.t } \sum_{k=1}^{K} \sum_{n=1}^{N} p_{k, n} \leq P_{T} \\
& \sum_{k=1}^{K} w_{k, n} \leq 1 \quad \forall n \\
& p_{k, n} \geq 0, \quad w_{k, n} \geq 0, \quad \forall k, \forall n,
\end{aligned}
$$

where $w_{k, n} \in\{0,1\}$. It means the $n$th subcarrier is allocated to the $k$ th user when $w_{k, n}$ is 1 . If $w_{k, n}$ is 0 , it means the $n$th subcarrier is not allocated to the $k$ th user. $P_{T}$ is the total transmit power. $P=\left(p_{k, n}\right)_{K \times N}, W=\left(w_{k, n}\right)_{K \times N}$.

\section{Karush-Kuhn-Tucker Theorem}

KKT conditions are proposed by Karush and Kuhn and Tucker independently. KKT conditions are first-order necessary conditions to solve optimization problem with equality and inequality constraints.

Consider the following maximization problem

$$
\begin{aligned}
\max & f(x) \\
\text { s.t } & g_{j}(x) \geq 0, \quad j=1,2, \ldots, m \\
& h_{i}(x)=0, \quad i=1,2, \ldots, n
\end{aligned}
$$

with $f: R^{N} \rightarrow R, g_{j}: R^{N} \rightarrow R^{p}, h_{i}: R^{N} \rightarrow R^{m}$ being continuously differentiable functions.

Kuhn-Tucker Theorem. If $x^{*}$ is a (local) optimum of the problem

$$
\begin{array}{cl}
\max & f(x) \\
\text { s.t } & g_{j}(x) \geq 0, \quad j=1,2, \ldots, m \\
& h_{i}(x)=0, \quad i=1,2, \ldots, n \\
\nabla f\left(x^{*}\right)+\nabla g\left(x^{*}\right) \lambda^{*}+\nabla h\left(x^{*}\right) \mu^{*}=0, \\
h\left(x^{*}\right)=0, \quad g\left(x^{*}\right) \geq 0, \\
\lambda^{*} \geq 0, \quad \lambda^{*} g\left(x^{*}\right)=0 .
\end{array}
$$

Equation (10) means $\nabla f$ is the linear combination of $\nabla g$ and $\nabla h$, where $\lambda^{*}$ and $\mu^{*}$ are called Lagrange multiplier. Equation (11) means the optimal point must meet all the constraints including equality and inequality constraints. That is to say the optimal point should be a feasible solution.

\section{Making Use of KKT Conditions}

For OFDM resource allocation problem, after the subcarrier assignment is completed, the above model becomes

$$
\begin{array}{ll}
\max & F(\bar{W}, P)=\sum_{k=1}^{K} \sum_{n=1}^{N} \frac{\bar{w}_{k, n}}{N} \ln \left(1+\frac{p_{k, n} h_{k, n}}{\Gamma}\right) \\
\text { s.t } & \sum_{k=1}^{K} \sum_{n=1}^{N} p_{k, n} \leq P_{T} \\
& p_{k, n} \geq 0, \quad k=1,2, \ldots, K, n=1,2, \ldots, N,
\end{array}
$$

where $\bar{W}, \bar{w}_{k, n}$ are known quantity. Equation (15) is a nonlinear equation, and it is continuous and differentiable. When $p_{k, n}>0, g_{k, n}=h_{k, n} / \Gamma$, a stagnation point of the equation can be obtained by KKT conditions:

$$
\begin{gathered}
\frac{\partial L}{\partial p_{k, n}}=\frac{g_{k, n}}{1+p_{k, n} g_{k, n}}-\lambda \leq 0 \\
p_{k, n}\left(\frac{g_{k, n}}{1+p_{k, n} g_{k, n}}-\lambda\right)=0, \quad k=1,2, \ldots, K, n=1,2, \ldots, N \\
\lambda \frac{\partial L}{\partial \lambda}=0
\end{gathered}
$$

Because $p_{k, n} \geq 0$, from (10), we can get

$$
\begin{aligned}
& \frac{g_{k, n}}{1+p_{k, n} g_{k, n}}-\lambda=0, \\
& \sum_{k=1}^{K} \sum_{n=1}^{N} p_{k, n}-P_{T}=0
\end{aligned}
$$

by $(12) \cdot p_{k, n}$ can be calculated by

$$
p_{k, n}(\bar{W})=\frac{P_{T}+\sum_{k=1}^{K}\left(\bar{w}_{k, n} / N\right) \sum_{n=1}^{N} g_{k, n}}{K \times N}-\frac{1}{g_{k, n}} .
$$

\section{Hybrid Evolutionary Algorithm for OFDM Resource Allocation}

Goldberg proposed the evolutionary algorithm's commonly form. The evolutionary algorithm (EA) simulates the natural selection in biological evolution. It is essentially a random search algorithm. EA's global search ability is excellent, and EA dose not demand the objective function continuous or differentiable. In (2), the variable $w_{k, n}$ is discrete, so EA can be used to solve subcarrier allocation. Meanwhile, KKT conditions are used to solve the variable $p_{k, n}$ by (14). Since the allocation of subcarrier and power is not independent, it is rational to combine these two algorithms to solve resource allocation problem.

For OFDM resource allocation, the two-step method cannot get a good solution because the allocation of subcarrier and power is not independent. In this paper, we propose 


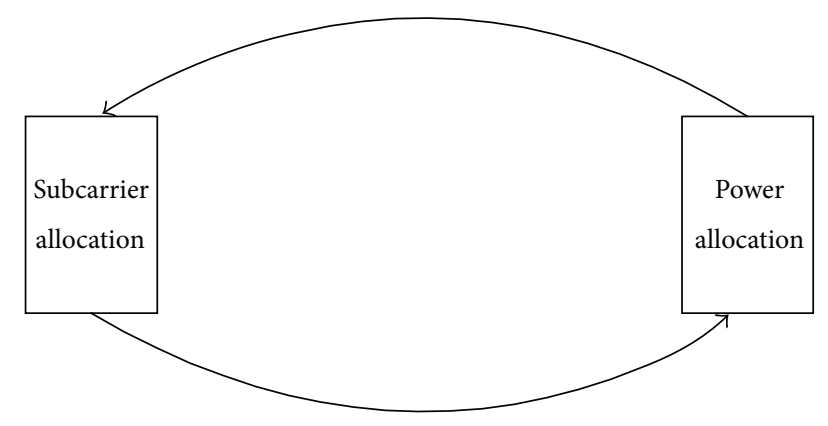

Figure 2: The information of optimizing subcarrier and power allocation can interact with each other.

a new resource allocation program based on KKT conditions. This new program combines evolutionary algorithm and KKT conditions. Firstly, we randomly generate some subcarrier allocation program $W_{1}, W_{2}, \ldots, W_{m}$. For each $W_{i}$, there is $F\left(W_{i}, P\right),(i=1,2, \ldots, m)$, and each $F\left(W_{i}, P\right)$ has only one variable $P . F\left(W_{i}, P\right)$ can be solved by (14). Assume the solution is $P_{i}$; for each $W_{i}$, we can get a $P_{i}$, $(i=1,2, \ldots, m)$. When $W_{i}$ and $P_{i}$ are known, we can get $F_{i} . F_{i}$ is the system rate corresponding to $W_{i}$. As previously described, $F_{i}$ is not a good result. Then, the proposed algorithm optimizes $W_{i},(i=1,2, \ldots, m)$ using evolutionary algorithm. Assume that $W_{1}(t), W_{2}(t), \ldots, W_{m}(t)$ is $t$ generation population of evolutionary algorithm; through (14), the $P_{1}(t), P_{2}(t), \ldots, P_{m}(t)$ can be calculated. Select individuals from $W_{1}(t), W_{2}(t), \ldots, W_{m}(t)$ to cross over and mutate. Then, new individuals are generated. Each new individual's power allocation can be calculated trough (14). According to every new individual's power allocation, select next generation individuals $W_{1}(t+1), W_{2}(t+1), \ldots, W_{m}(t+1)$. So, the information of power and subcarrier allocation can make full use of each other. It is described in Figure 2.

5.1. Fitness Function. A hybrid evolutionary algorithm for OFDM resource allocation is proposed in this paper. This algorithm firstly generates a set of subcarrier allocation programs $W_{1}, W_{2}, \ldots, W_{m}$, where $W_{i}$ is $i$ th subcarrier allocation program, $i=1,2, \ldots, m$. For each $W_{i}$, its corresponding power allocation $p_{i}$ can be obtained through (14), then, the fitness function of $W_{i}=\left(w_{k, n}^{i}\right)_{K \times N}$ can be calculated as follows:

$$
F\left(W_{i}, P\right)=\sum_{k=1}^{K} \sum_{n=1}^{N} \frac{w_{k, n}^{i}}{N} \ln \left(1+\frac{p_{k, n} h_{k, n}}{\Gamma}\right) .
$$

5.2. Encoding of Solutions. Evolutionary algorithm has attracted more and more attentions because of its good performance in the engineering field. For evolutionary algorithm, encoding is one of the most important parts in EA. An excellent encoding method cannot only avoid generating illegal solutions but also improve the performance of the algorithm.
The subcarrier allocation program $W$ is a $K \times N$ matrix as follows:

$$
W=\left(\begin{array}{ccccccc}
1 & 0 & 0 & \ldots & 0 & 1 & 0 \\
0 & 1 & 1 & \ldots & 0 & 0 & 1 \\
0 & 0 & 0 & \ldots & 1 & 0 & 0 \\
0 & 0 & 0 & \ldots & 0 & 0 & 0 \\
& & \ldots & \ldots & \ldots & & \\
0 & 0 & 0 & \ldots & 0 & 0 & 0
\end{array}\right) .
$$

It is clear that there is only one 1 in each column, which means each subcarrier can only be allocated to one user. When $K$ and $N$ are very large, the computational process expends a lot of time. What is more, the process of crossover and mutation would be very complex. So, it is necessary to encode $W$ for simple operation. In this paper, $W$ is encoded as follows:

$$
W=\left(w_{1}, w_{2}, \ldots, w_{N}\right),
$$

where $w_{i}$ indicates the $i$ th subcarrier allocated to $w_{i}$ th user. For example, if $w_{i}$ is 5 , it means the $i$ th subcarrier is allocated to the 5 th user.

$P$ is also a $K \times N$ matrix as follows:

$$
P=\left(\begin{array}{ccccc}
p_{1,1} & p_{1,2} & p_{1,3} & \ldots & p_{1, N} \\
p_{2,1} & p_{2,2} & p_{2,3} & \ldots & p_{2, N} \\
& \ldots & \ldots & \ldots & \\
& \ldots & \ldots & \ldots & \\
\ldots & \ldots & \ldots & \\
p_{K, 1} & p_{K, 2} & p_{K, 3} & \ldots & p_{K, N}
\end{array}\right),
$$

where $p_{i, j}$ is the power of the $j$ th subcarrier allocated to $i$ th user. When $W$ is known, $P$ can be simplified into

$$
P=\left(p_{1}, p_{2}, \ldots, p_{N}\right),
$$

where $p_{i}$ is the power of $i$ th subcarrier. For example, if $p_{i}=$ 0.5 , it means the $i$ th subcarrier's power is $0.5 \mathrm{w}$.

5.3. The Proposed Algorithm's Framework. The hybrid evolutionary algorithm proposed in this paper firstly generates a set of subcarrier allocation programs $W_{1}, W_{2}, W_{3}, \ldots, W_{m}$. Then, we can obtain the power allocation program corresponding to each subcarrier allocation program from (14). Substituting $W_{i}$ and $P_{i}$ in (14), we can get each individual's fitness $F\left(W_{i}, P_{i}\right)$ of $W_{i}$. These subcarrier allocation programs are regarded as a population, and each subcarrier allocation program is an individual. The fitness of each individual is the system rate obtained by (15). After crossover and mutation, we can get a new population constituted by new subcarrier allocation programs. A new power allocation program and system rate can be calculated. Repeat the above steps; the information optimizing power and the subcarrier allocation can interact with each other. Therefore, the performance of the algorithm is improved greatly. According to the above description, the framework of hybrid evolutionary algorithm for OFDM resource allocation is as follows.

\section{The Proposed Algorithm}

Step 1. Generate a set of subcarrier allocation programs $\left\{W_{1}, W_{2}, \ldots, W_{m}\right\}$ as the initial population, denoted as pop. 


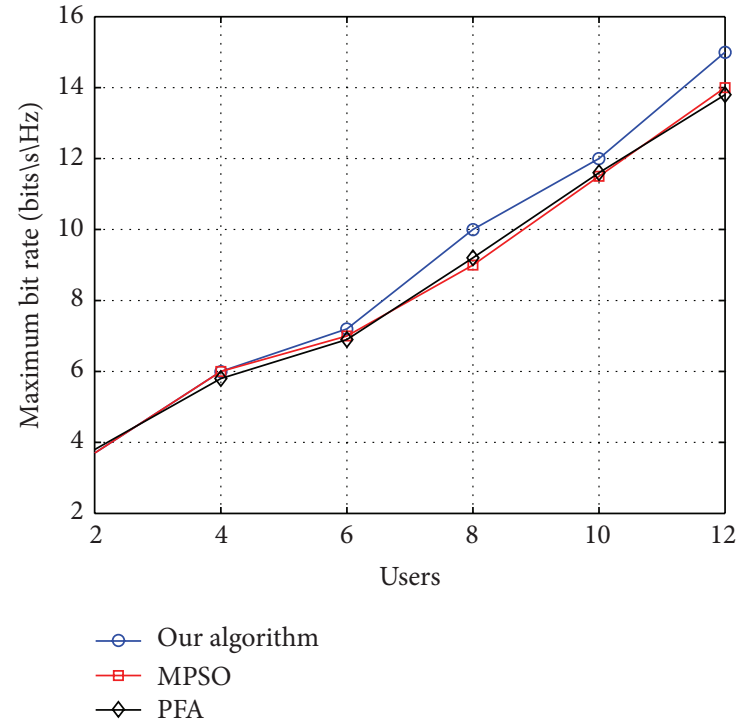

FIGURE 3: System throughput versus number of users.

Step 2. Calculate the fitness of each individual according to (15).

Step 3. Select individuals from pool to crossover, and generate offspring population, denoted as child.

Step 4. Select individuals from child to mutate in mutation probability and update child.

Step 5. Renew pop with child.

Step 6. If the stopping criteria are unsatisfied, go to Step 2; otherwise, stop.

\section{Computer Simulation}

We compare the proposed algorithm with $[10,25]$ in the aspects of the transmit power and system capacity in case the number of users changes from 2 to 12 . In the numerical simulations, the channel is modeled as slow-varying Rayleigh and its components have independent identically distributed complex values with zero-mean and unit variance. Total transmit power, BER, and the total bandwidth are set as $0.1 \mathrm{w}$, $10^{-3}$, and $1 \mathrm{MHZ}$, respectively. The number of subcarrier is 64 , and the crossover probability and mutation probability are 0.8 and 0.01 .

Figure 3 shows the performance of the maximum data rate with growth of the number of users. Figure 4 also shows that the system total rate calculated by the three algorithms is almost the same when the number of users is less. However, as the number of users increases, the performance of the algorithm presented by this paper is better than that of the other two algorithms. Then, we test the proposed algorithm's minimum transmit power and compare it with MPSO [25] and PFA [10]. As it is shown in Figure 4, when the number of users is less than 4 , the proposed algorithm's performance is not better than that of the other two algorithms. However,

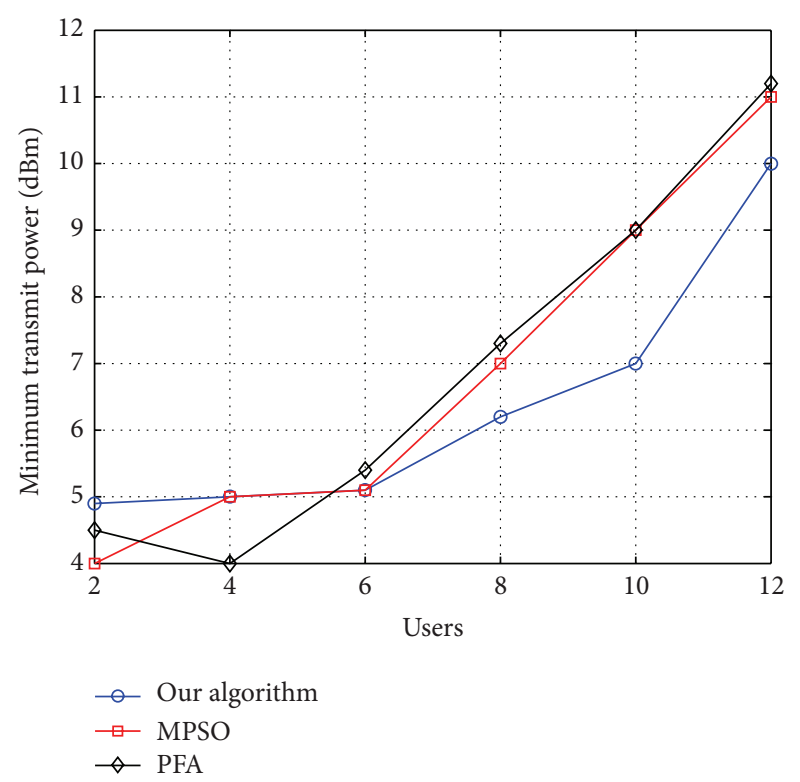

Figure 4: Minimum transmit power versus number of users.

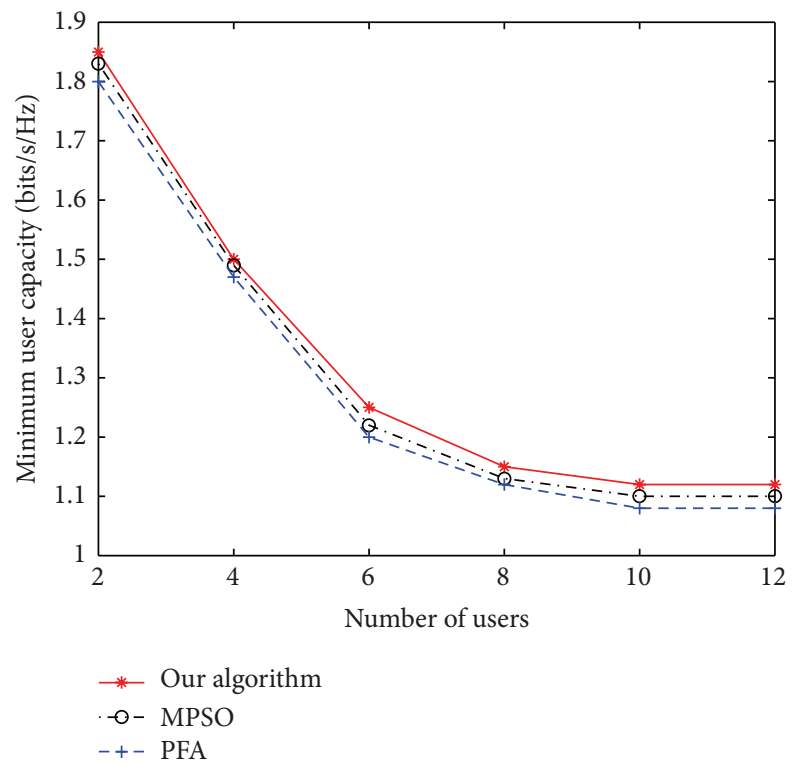

FIGURE 5: minimum user capacity for multiuser OFDM versus number of users for proposed algorithm and other methods.

since the proposed algorithm combines evolutionary algorithm with KKT conditions, it becomes better than MPSO and PFA with the users number increasing. It is clear that the proposed algorithm has superior performance.

Figure 5 shows the minimum user capacity of the proposed algorithm, MPSO, and PFA with growth of the total users number. If the minimum user capacity is increased, the total system rate can also increase. From Figure 5, MPSO's minimum user capacity is much larger than that of PFA, and the proposed algorithm's minimum user capacity is much larger than MPSO. 


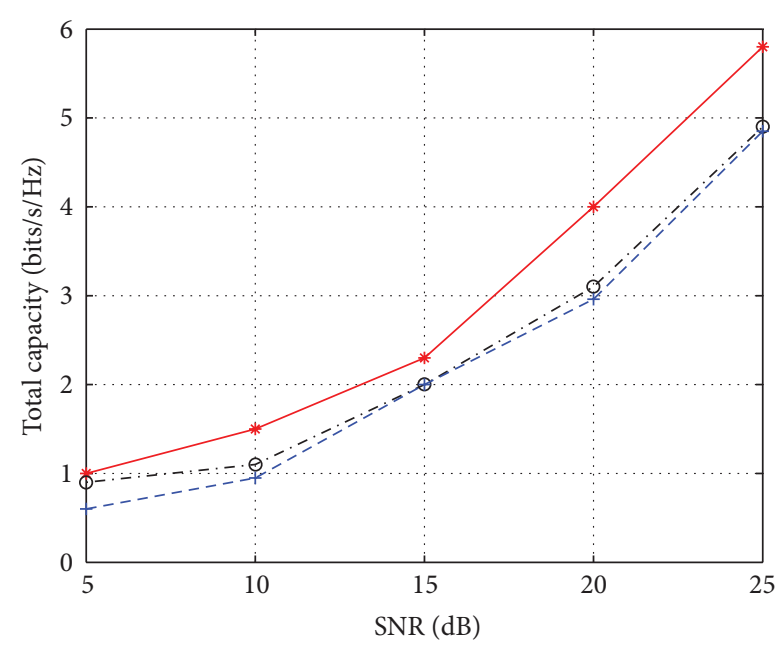

$$
\begin{aligned}
& \text { *- Our algorithm } 4 \text { users } \\
& \text { - - MPSO } 4 \text { users } \\
& \text {-+- PFA } 4 \text { users }
\end{aligned}
$$

FIGURE 6: Capacity comparison of our algorithm and other two algorithms as the distributions of 4 users are different.

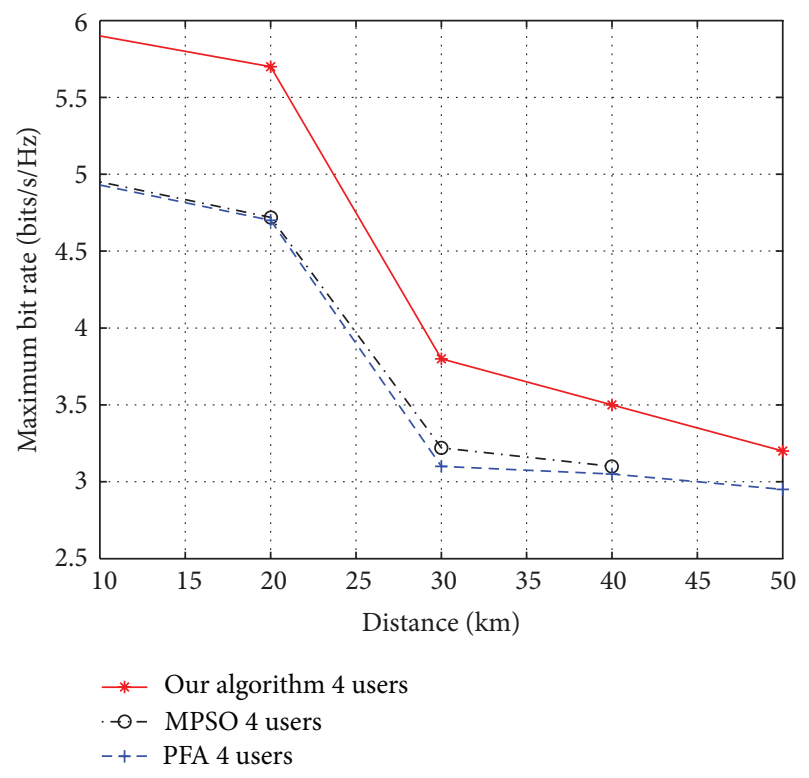

FIGURE 7: Capacity comparison of our algorithm and another two algorithms with different distance.

Then, this paper compares the capacity of the proposed algorithm, MPSO, and PFA under different SNR with 4 users. The comparison result is shown in Figure 6. From Figure 6, it is clear that the proposed algorithm's performance is better than the other two algorithms. When SNR is less than $15 \mathrm{~dB}$, the performance of the proposed algorithm improves slowly, although it is also better than the other two algorithms. With the increasing of SNR, the proposed algorithm's performance enhances more and more fast. Figure 8 shows the minimum transmit power of the proposed algorithm, MPSO, and PFA under different SNR with 8 users. From Figure 8, the

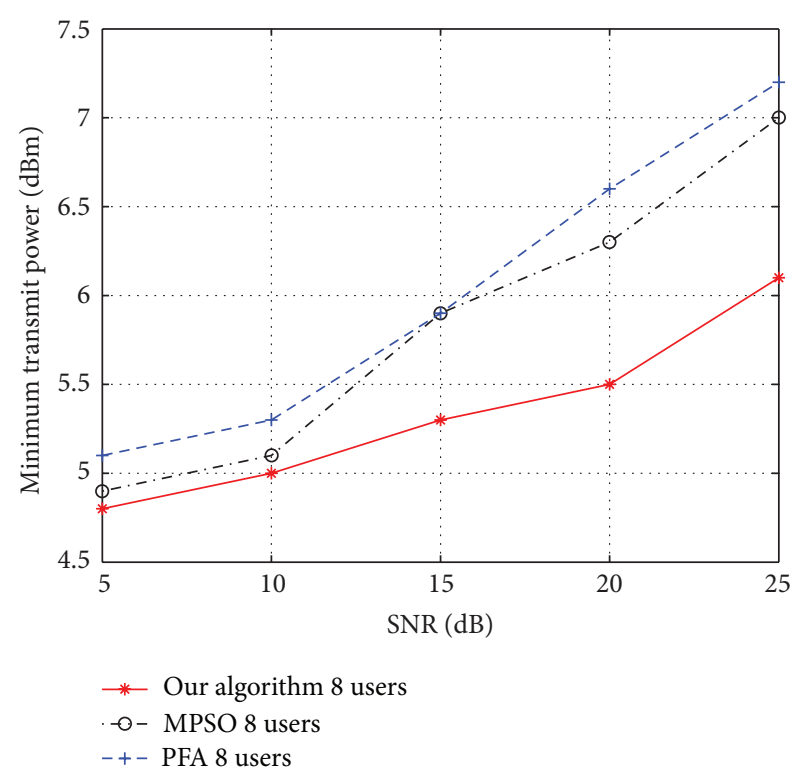

FIGURE 8: Minimum transmit power of our algorithm and another two algorithms as the distributions of 8 users are different.

TABLE 1

\begin{tabular}{lccc}
\hline Run index & Proposed algorithm & MPSO & PFA \\
\hline 1 & 1.407 & 3.012 & 1.112 \\
2 & 1.507 & 3.105 & 1.203 \\
3 & 1.426 & 3.125 & 1.235 \\
4 & 1.620 & 3.106 & 1.135 \\
5 & 1.526 & 2.988 & 1.125 \\
6 & 1.458 & 3.241 & 1.109 \\
7 & 1.635 & 3.075 & 1.246 \\
8 & 1.425 & 3.005 & 1.106 \\
9 & 1.326 & 3.276 & 1.011 \\
10 & 1.427 & 3.006 & 1.189 \\
\hline
\end{tabular}

TABLE 2: System rate variance for 100 experiments.

\begin{tabular}{lccc}
\hline Algorithm & 2 users & 4 users & 6 users \\
\hline Proposed algorithm & 0.00000 & 0.00060 & 0.00150 \\
MPSO & 0.00000 & 0.00220 & 0.00760 \\
PFA & 0.00000 & 0.00120 & 0.00550 \\
\hline
\end{tabular}

proposed algorithm's minimum transmit power is less than that of the other two algorithms.

Figure 7 shows the capacity comparison of the proposed algorithm and the other two algorithms with different distance, and the number of user is 4 . From Figure 7, the total capacity is reducing with the distance becoming longer and longer. And the proposed algorithm shows a better performance against the increase of distance.

In Table 1, we calculate the execution time to reach the target capacity needed by the proposed algorithm, MPSO, and $\mathrm{PFA}$, respectively. The target capacity is preset to $14 \mathrm{bit} / \mathrm{s} / \mathrm{Hz}$ with 12 users. It is clear that the proposed algorithm can reach the target capacity more fast. It is more efficient to run the 
TABLE 3: System rate variance for 100 experiments.

\begin{tabular}{lccc}
\hline Algorithm & 8 users & 10 users & 12 users \\
\hline Proposed algorithm & 0.00230 & 0.00700 & 0.00960 \\
MPSO & 0.00940 & 0.01200 & 0.01820 \\
PFA & 0.00770 & 0.00850 & 0.00920 \\
\hline
\end{tabular}

TABLE 4: Minimize power variance for 100 experiments.

\begin{tabular}{lccc}
\hline Algorithm & 2 users & 4 users & 6 users \\
\hline Proposed algorithm & 0.00000 & 0.00000 & 0.00080 \\
MPSO & 0.00000 & 0.00060 & 0.00240 \\
PFA & 0.00000 & 0.00000 & 0.00120 \\
\hline
\end{tabular}

TABle 5: Minimize power variance for 100 experiments.

\begin{tabular}{lccc}
\hline Algorithm & 8 users & 10 users & 12 users \\
\hline Proposed algorithm & 0.00085 & 0.00090 & 0.00130 \\
MPSO & 0.00270 & 0.00320 & 0.00390 \\
PFA & 0.00160 & 0.00200 & 0.00340 \\
\hline
\end{tabular}

proposed algorithm than to run other two algorithms. It is mainly because the proposed algorithm optimizes subcarrier and power allocation at the same time, and in this algorithm, the information of subcarrier and power allocation can interact with each other. So, the proposed algorithm's convergence is better.

Tables 2 and 4 are system rate variance for 100 experiments and minimize power variance for 100 experiments, respectively, when the users number changes from 2 to 6 . Tables 3 and 5 are system rate variance for 100 experiments and minimizing power variance for 100 experiments respectively, when the users number changes from 8 to 12 . They reflect the the stability of algorithm.

These four tables show the stability of the proposed algorithm is similar to PFA and is better than MPSO in the case of 2 users and 4 users. In the case of more than 6 users, the stability of our proposed algorithm is better than that of MPSO and PFA.

\section{Conclusions}

Since the subcarrier and power allocation are dependent, it is reasonable to optimize them at the same time. However, because the subcarrier allocation is a discrete problem and the power allocation is a continuous problem, it is very difficult to optimize subcarrier and power allocation at the same time. This paper proposes a hybrid evolutionary algorithm for OFDM resource allocation. This algorithm can optimize subcarrier allocation and transmit power alternately. The process optimizing power allocation can take advantage of the information of subcarrier allocation, and the process optimizing subcarrier allocation can also take advantage of the information of power allocation. Therefore, the information optimizing power and subcarrier allocation can interact with each other, and the efficiency of the algorithm can be improved significantly. The computer simulation shows that the proposed algorithm increases the system rate apparently.

\section{Acknowledgments}

This work was supported in part by the Natural Science Foundation of China (60974077), the Natural Science Foundation of Guangdong Province (S2011030002886, S2012010008813), Programme of Science and Technology of Guangdong Province (2012B091100033), Programme of Science and Technology of the Department of Education of Guangdong Province (2012KJCX0042), and Zhongshan Programme of science and technology (20114A223).

\section{References}

[1] S. Sadr, A. Anpalagan, and K. Raahemifar, "Radio resource allocation algorithms for the downlink of multiuser OFDM communication systems," IEEE Communications Surveys and Tutorials, vol. 11, no. 3, pp. 92-106, 2009.

[2] H. Seo and B. G. Lee, "A proportional-fair power allocation scheme for fair and efficient multiuser OFDM systems," in Proceedings of the IEEE Global Telecommunications Conference (GLOBECOM '04), pp. 3737-3741, Dallas, Tex, USA, December 2004.

[3] J. Huang, V. Subramanian, R. Agrawal, and R. Berry, "Joint scheduling and resource allocation in uplink OFDM systems for broadband wireless access networks," IEEE Journal on Selected Areas in Communications, vol. 27, no. 2, pp. 226-234, 2009.

[4] D. Wang, Z. Li, and X. Wang, "Joint optimal subcarrier and power allocation for wireless cooperative networks over OFDM fading channels," IEEE Transactions on Vehicular Technology, vol. 61, no. 1, pp. 249-257, 2012.

[5] J. Huang, V. Subramanian, R. Agrawal, and R. Berry, "Joint scheduling and resource allocation in uplink OFDM systems for broadband wireless access networks," IEEE Journal on Selected Areas in Communications, vol. 27, no. 2, pp. 226-234, 2009.

[6] A. Maiga, J. Y. Baudais, and J. F. Hélard, "Subcarrier, bit and time slot allocation for multicast precoded OFDM systems," in Proceedings of the IEEE International Conference on Communications (ICC '10), pp. 1-6, May 2010.

[7] X. Wang and G. B. Giannakis, "Resource allocation for wireless multiuser OFDM networks," IEEE Transactions on Information Theory, vol. 57, no. 7, pp. 4359-4372, 2011.

[8] A. N. Zaki and A. O. Fapojuwo, "Optimal and efficient graphbased resource allocation algorithms for multiservice framebased OFDMA networks," IEEE Transactions on Mobile Computing, vol. 10, no. 8, pp. 1175-1186, 2011.

[9] W. W. L. Ho and Y. C. Liang, "Optimal resource allocation for multiuser MIMO-OFDM systems with user rate constraints," IEEE Transactions on Vehicular Technology, vol. 58, no. 3, pp. 1190-1203, 2009.

[10] T. D. Nguyen and Y. Han, "A proportional fairness algorithm with QoS provision in downlink OFDMA systems," IEEE Communications Letters, vol. 10, no. 11, pp. 760-762, 2006.

[11] A. S. Mahmoud, A. Y. Al-Rayyah, and T. R. Sheltami, "Adaptive power allocation algorithm to support absolute proportional rates constraint for scalable OFDM systems," in Proceedings of the 71st IEEE Vehicular Technology Conference (VTC '10), pp. 14, Taipei, Taiwan, May 2010. 
[12] M. S. Maw and I. Sasase, "Efficient resource allocation for multiuser MIMO-OFDM Uplink system to guarantee the proportional data rate fairness among users in a system," in Proceedings of the 1st International Symposium on Access Spaces (ISAS '11), pp. 132-137, Yokohama, Japan, 2011.

[13] Y. Li, N. Lu, M. Peng, and W. Wang, "Multiuser resource allocation for OFDM downlink with terminal bandwidth limitation," in Proceedings of the IEEE Wireless Communications and Networking Conference (WCNC '10), pp. 2378-2390, April 2010.

[14] P. D. Morris and C. R. N. Athaudage, "Fairness based resource allocation for multi-user MIMO-OFDM systems," in Proceedings of the 63rd IEEE Vehicular Technology Conference (VTC '06), pp. 314-318, July 2006.

[15] A. Fahmi, M. Asvial, and D. Gunawan, "Uplink resource allocation algorithms with fractional power control as power constraints for OFDMA system," in Proceedings of the IEEE Region 10 Conference (TENCON '11), pp. 592-596, 2011.

[16] H.-G. Chen, L.-P. Lu, and M.-Y. Zhai, "A new OFDM resource allocation algorithm based on the bit exchange scheme," in Proceedings of the IEEE International Conference on Oxide Materials for Electronic Engineering, pp. 837-840, 2012.

[17] Z. Han, T. Himsoon, W. P. Siriwongpairat, and K. J. R. Liu, "Resource allocation for multiuser cooperative OFDM networks: who helps whom and how to cooperate," IEEE Transactions on Vehicular Technology, vol. 58, no. 5, pp. 23782391, 2009.

[18] K. D. Lee and T. S. P. Yum, "On pareto-efficiency between profit and utility in OFDM resource allocation," IEEE Transactions on Communications, vol. 58, no. 11, pp. 3277-3285, 2010.

[19] M. Li, X. Wang, H. Zhang, and M. Tang, "Resource allocation with subcarrier cooperation in OFDM-based wireless multicast system," in Proceedings of the 73rd IEEE Vehicular Technology Conference (VTC '11), pp. 1-5, Budapest, Hungary, 2011.

[20] X. Zhao, L. Zheng, M. Huang, and J. Wang, "Proportional resource allocation for multi-user OFDM systems," in Proceedings of the IEEE International Conference on Information Theory and Information Security (ICITIS '10), pp. 1100-1103, December 2010.

[21] D. Perea-Vega, J. F. Frigon, and A. Girard, "Near-optimal and efficient heuristic algorithms for resource allocation in MISO-OFDM systems," in Proceedings of the IEEE International Conference on Communications (ICC '10), pp. 1-6, Cape Town, South Africa, May 2010.

[22] Q. Wang, H. Liu, and J. Li, "Resource allocation for OFDM system based on evolutionary algorithm," in Proceedings of the 8th International Conference on Wireless Computational Intelligence and Security (CIS '12), pp. 77-81, 2012.

[23] N. Ksairi, P. Bianchi, P. Ciblat, and W. Hachem, "Resource allocation for downlink cellular OFDMA systems-part II: practical algorithms and optimal reuse factor," IEEE Transactions on Signal Processing, vol. 58, no. 2, pp. 735-749, 2010.

[24] N. A. Odhah, M. I. Dessouky, W. E. Al-Hanafy, and F. E. A. El-Samie, "Greedy power allocation algorithm for proportional resource allocation in multi-user OFDM systems," in Proceedings of the 29th National Radio Science Conference (NRSC '12), pp. 421-428, Cairo, Egypt, 2012.

[25] S. Sadeque, I. Ahmed, and R. Vaughan, "Impact of individual and joint optimizations in multi-user OFDM resource allocation by modified PSO," in Proceedings of the 24th Canadian Conference on Electrical and Computer Engineering (CCECE '11), pp. 001233-001237, Niagara Falls, Canada, 2011.
[26] R. Annauth and H. C. S. Rughooputh, "Evolutionary multiobjective approach for resource allocation in OFDM systems," in Proceedings of the 4th International Joint Conference on Computational Sciences and Optimization, pp. 195-199, 2011.

[27] I. Ahmed and S. P. Majumder, "Adaptive resource allocation based on modified genetic algorithm and particle swarm optimization for multiuser OFDM systems," in Proceedings of the 5th International Conference on Electrical and Computer Engineering (ICECE '08), pp. 211-216, December 2008. 


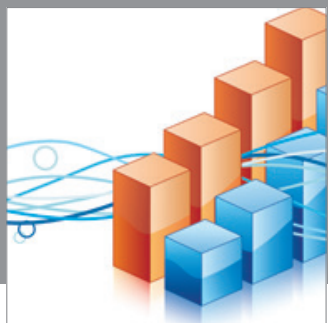

Advances in

Operations Research

mansans

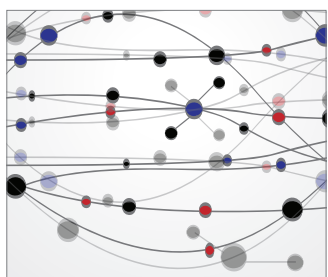

The Scientific World Journal
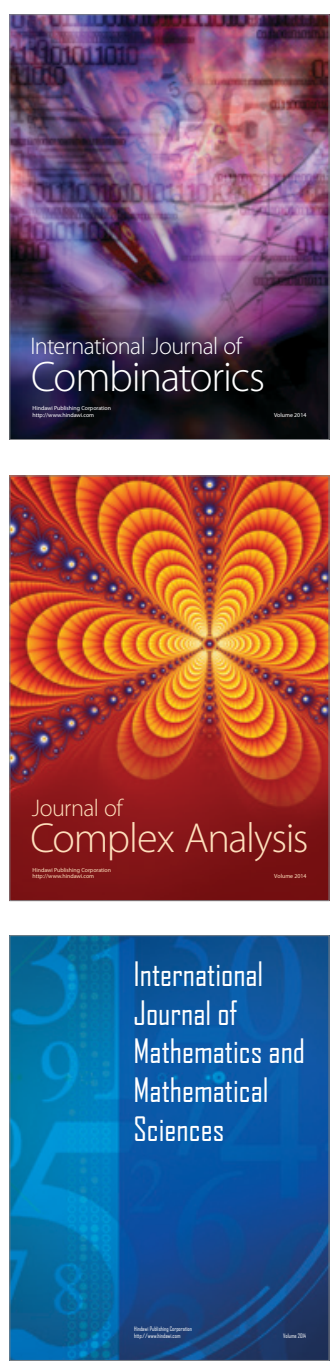
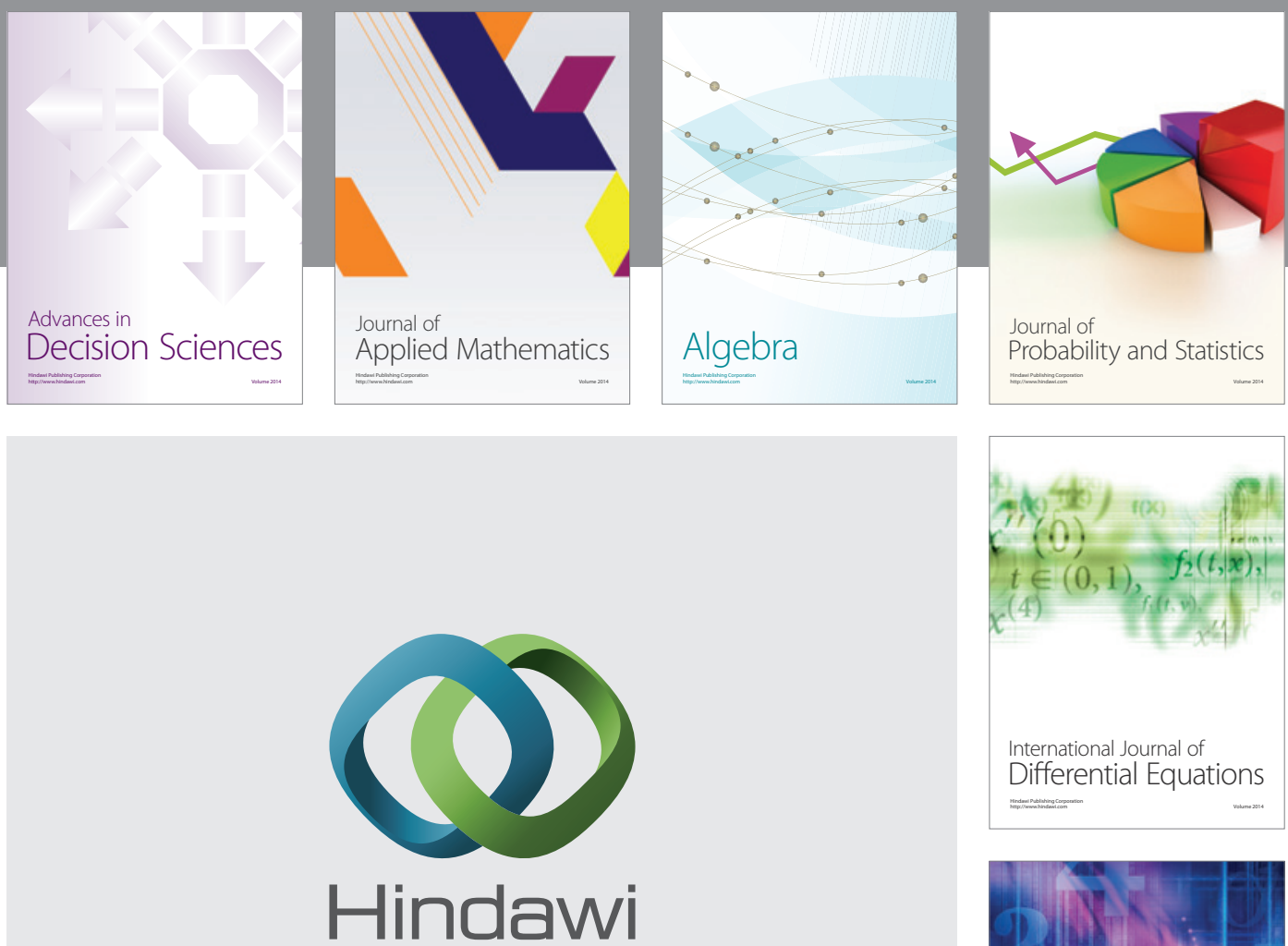

Submit your manuscripts at http://www.hindawi.com
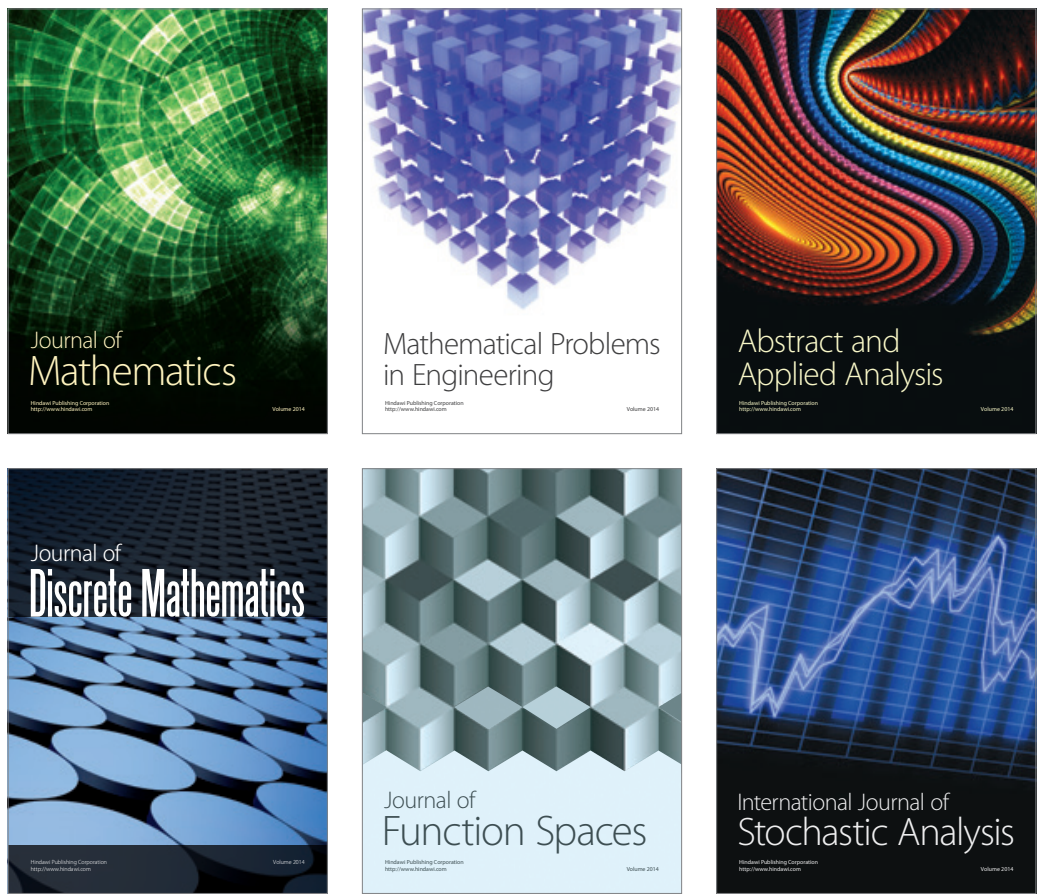

Journal of

Function Spaces

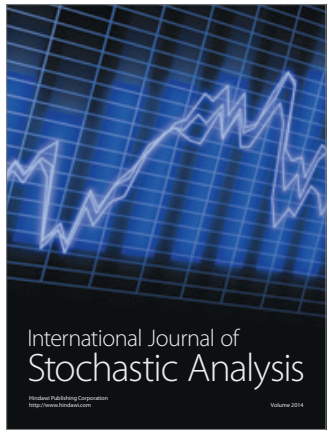

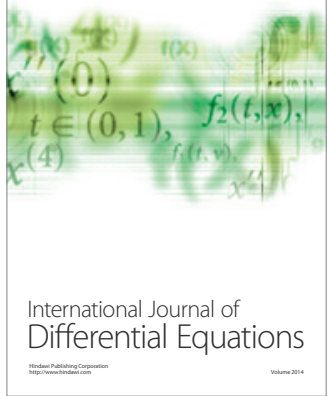
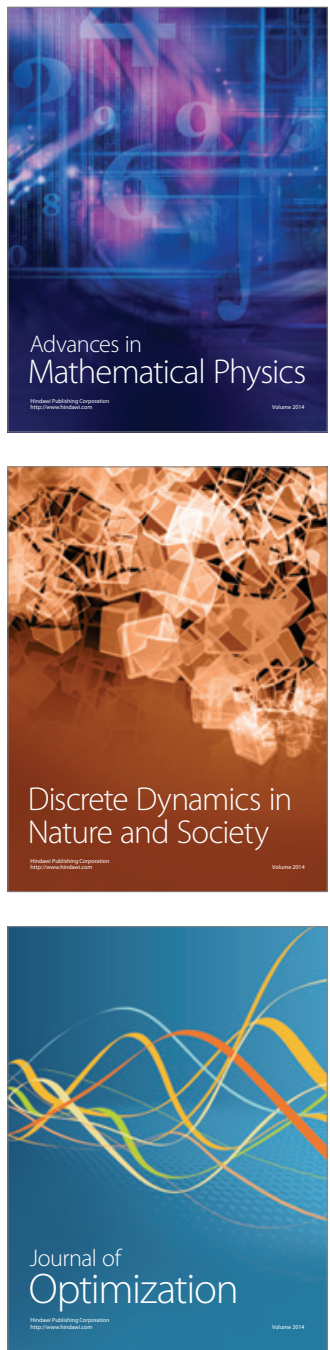